\title{
Body composition and plasma levels of inflammatory biomarkers in COPD
}

\author{
T.M.L. Eagan*,\#, P. Aukrust ${ }^{\uparrow,+}$, T. Ueland ${ }^{\uparrow, \S}$, J.A. Hardie*, A. Johannessen ${ }^{f}$,

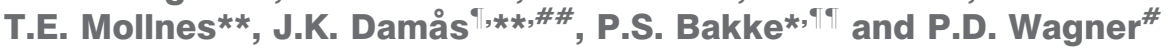

ABSTRACT: Previous studies suggest a relationship between systemic inflammation and body composition in chronic obstructive pulmonary disease (COPD). We examined the relationships between body composition (fat free mass index (FFMI) $\mathrm{kg} \cdot \mathrm{m}^{-2}$ and fat mass index (FMI) $\mathrm{kg} \cdot \mathrm{m}^{-2}$ ) and three plasma inflammatory markers C-reactive Protein (CRP), soluble tumour necrosis factor receptor 1 (sTNF-R1) and osteoprotegerin (OPG) in 409 stable COPD patients (aged 40-75 yrs, Global Initiative for Obstructive Chronic Lung Disease (GOLD) categories II-IV, 249 male) from the Bergen COPD Cohort Study in Norway.

FFMI and FMI were measured by bioelectrical impedance. Plasma CRP $\left(\mu \mathrm{g} \cdot \mathrm{mL}^{-1}\right)$, sTNF-R1 $\left(\mathrm{pg} \cdot \mathrm{mL}^{-1}\right)$ and OPG $\left(\mathrm{ng} \cdot \mathrm{mL}^{-1}\right)$ were determined by enzyme immunoassays. Correlations and Kruskal-Wallis tests were used for bivariate analyses. Linear regression models were fitted for each of the three markers, CRP, sTNF-R1 and OPG, with FFMI and FMI as explanatory variables including sex, age, smoking habits, GOLD category, hypoxaemia, Charlson Comorbidity Index and inhaled steroid use as potential confounders.

CRP and sTNF-R1 levels correlated positively with both FFMI and FMI. The adjusted regression coefficients for an increase in logCRP per unit increase in FFMI was $1.23(1.14-1.33) \mathrm{kg} \cdot \mathrm{m}^{-2}$ and $24.9(11.8-38.1) \mathrm{kg} \cdot \mathrm{m}^{-2}$ for sTNF-R1. Higher FMI was associated with a lower OPG, with adjusted regression coefficient $-0.14(-0.23--0.04)$, whereas FFMI was unrelated to OPG.

In conclusion, COPD patients with low FFMI had lower not higher plasma levels of CRP and sTNF-R1, whereas higher fat mass was associated with higher CRP and sTNF-R1 and lower OPG.

KEYWORDS: Cachexia, chronic obstructive pulmonary disease, C-reactive protein, inflammation

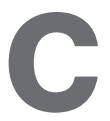
hronic obstructive pulmonary disease (COPD) is a chronic inflammatory disease of the airways and lung parenchyma, estimated to affect $9-10 \%$ of adults aged $>40$ yrs [1]. COPD causes both significant mortality [2] and morbidity [3].

Approximately $25 \%$ of patients with COPD experience a pathological loss of lean body mass [4]. COPD patients with abnormally low lean body mass are at an increased risk of death compared to those with normal lean body mass [5]. The term cachexia is often used to describe the state of pathologically low lean body mass. However, some authors define cachexia as a state in which protein is lost specifically due to proinflammatory processes, such as those driven by tumour necrosis factor (TNF)- $\alpha$ and interleukin (IL)-6 [6]. Although an increase in systemic inflammation is arguably the prevailing theory for loss of lean body mass in some COPD patients, this theory remains unproven [4].

Support for the role of systemic inflammation comes from cross-sectional studies in which higher blood levels of TNF- $\alpha$ [7-10], IL-6 [9, 10] and C-reactive protein (CRP) [9] were seen in underweight COPD patients compared with normal weight patients. However, in earlier studies [7-9], body mass index (BMI) was used to stratify patients. This is imperfect, since patients can have significant muscle wasting without a significant decrease in total body weight, if there is a corresponding increase in fat mass [11].

Four later studies have included measurements of fat-free mass index (FFMI) by bioelectrical impedance [12-15]. Among these studies there was no statistically significant relationship between levels of TNF- $\alpha$ and body composition in the three studies which measured TNF- $\alpha$ [1214]. In the fourth study, CRP was negatively associated with FFMI in bivariate analyses, but not after adjustment for confounder age, number of lymphocytes, handgrip strength, walking distance on 6-min walk test and per cent lowattenuating area on high-resolution computed tomography scans [15]. The total number of

\section{AFFILIATIONS}

${ }^{*}$ Dept of Thoracic Medicine,

${ }^{f}$ Centre for Clinical Research Haukeland University Hospital, "Section of Pulmonary Medicine, Institute of Medicine, University of Bergen, Bergen,

"Research Institute for Internal Medicine, Rikshospitalet University Hospital, University of Oslo,

+Section of Clinical Immunology and Infectious Diseases, Rikshospitalet University Hospital, University of Oslo,

${ }^{\S}$ Section of Endocrinology, Rikshospitalet University Hospital, University of Oslo,

${ }^{* *}$ Institute of Immunology, Rikshospitalet University Hospital, University of Oslo, Oslo and \#\#Dept of Infectious Diseases, St. Olavs Hospital, Trondheim, Norway, \#Division of Physiology, University of California San Diego, La Jolla, CA, USA.

CORRESPONDENCE

T.M.L. Eagan

Dept of Thoracic Medicine Haukeland University Hospital Jonas Lies vei

5021 Bergen

Norway

E-mail: tomas.eagan@med.uib.no

Received:

Dec 082009

Accepted after revision:

April 042010

First published online:

April 222010

European Respiratory Journal Print ISSN 0903-1936 Online ISSN 1399-3003 
COPD patients in these studies was generally small and varied between 20 subjects [13] and 99 subjects [12].

Because of confounding variables there is still considerable uncertainty regarding the question of whether COPD patients with low FFMI have different blood levels of important inflammatory markers or not. To shed further light on this question, it is necessary to have a sample size that allows for multivariate analysis. The Bergen COPD Cohort Study has enrolled $>400$ COPD patients and is powered for a multivariate analysis. It has, therefore, provided an opportunity to assess the relationship between pro-inflammatory markers and body composition defined by both FFMI and fat mass index (FMI). We have recently published the levels of six inflammatory markers in COPD patients and subjects without COPD [16]. Of these, CRP, soluble TNF receptor 1 (sTNF-R1) and osteoprotegerin (OPG) were potentially related to body composition in COPD based on previous studies for CRP and STNF-R1 [7-11, 13-15, 17], and for OPG based on its role in bone metabolism [18] and in metabolic disorders like diabetes [19]. Thus, our study reports the relationship between these three markers and body composition in the $>400$ COPD patients at entry into the Bergen COPD Cohort Study.

\section{METHODS}

\section{Study population}

The first phase of the Bergen COPD Cohort study was undertaken from 2006 to 2007, and the selection of the study population and data collection has recently been published in greater detail [16]. Altogether 433 COPD patients from western Norway, aged 40-75 yrs, were included in the first phase of the Bergen COPD Cohort study. Approximately $75 \%$ of the COPD patients were recruited through the outpatient clinic of the Dept of Thoracic Medicine (Haukeland University Hospital, Bergen, Norway). The remaining 25\% were recruited from surrounding hospitals in western Norway, or from referrals from three private lung physicians outside the hospital.

All patients had a clinical diagnosis of COPD, a smoking history of $>10$ pack-yrs, a post-bronchodilator forced expiratory volume in $1 \mathrm{~s}(\mathrm{FEV} 1) /$ forced vital capacity ratio $<0.7$, and an FEV1of $<80 \%$ predicted based on Norwegian pre-bronchodilation reference values [20]. Patients with known cancer in the last 5 yrs or with a known inflammatory disorder such as rheumatoid arthritis, systemic lupus erythematosus or inflammatory bowel disease were not included. Common chronic comorbid disorders with known inflammatory components, such as coronary heart disease, hypertension and diabetes, were not cause for exclusion. No COPD patients were included if judged by the study physician to have had an exacerbation in the last 4 weeks prior to enrolment.

All patients received written information prior to participation, and all patients signed informed consent. The regional ethical committee (REK Vest, Bergen, Norway) approved the study.

\section{Data collection}

A physician examined all patients and recorded a full medical history including smoking history and comorbidities. The physician performed arterial blood gas sampling and immediately analysed the sample on a Radiometer ABL 520 blood gas analyser (Radiometer, Copenhagen, Denmark). Lung function measurements were performed with a Viasys Masterscope (Viasys, Hoechburg, Germany) before and 15 min after an inhalation of $0.4 \mathrm{mg}$ salbutamol. Bioelectrical impedance was used to measure fat-free mass after overnight fasting using a Bodystat 1500 (Bodystat Ltd, Isle of Man, UK). The FFMI was defined as the fat free mass $(\mathrm{kg})$ divided by the square of height $\left(\mathrm{m}^{2}\right)$, and similarly for the FMI.

Plasma samples were centrifuged within $30 \mathrm{~min}$ at 2,150 $\times g$ $\left(15 \mathrm{~min}\right.$ at $\left.4^{\circ} \mathrm{C}\right)$. All samples were stored at $-80^{\circ} \mathrm{C}$ and thawed less three times. The inflammatory markers CRP, sTNF-R1 and OPG were analysed by enzyme immunoassays (EIAs), as previously described [16].

\section{Statistical analyses}

Stata 10.1 (Stata Statistical Software, College Station, TX, USA) was used for computation. Based on medical history, Charlsons Comorbidity Index was calculated [21, 22]. Since females and males differ in body composition with regard to fat mass, muscle mass and bone density, quartiles of FFMI and FMI were calculated for females and males separately, and then pooled into one variable for both sexes for FFMI and FMI quartiles respectively. Bivariate associations between the levels of each of the three markers and FFMI and FMI in quartiles were tested with the Kruskal-Wallis nonparametric test. For the multivariate analyses, linear multivariable regression was performed with the three markers as the outcome variables. Due to the non-normal distribution of the residuals, logCRP was used as the outcome in the regression analysis examining the effects of FFMI and FMI on CRP. The independent variables studied were sex, age, smoking (ex- versus current smoker), FEV1 \% pred, hypoxaemia, Charlson Comorbidity Index and use of inhaled steroids. Multicollinarity prevented the simultaneous inclusion of FFMI and FMI directly in the multivariable regression models. Instead, the method of residualisation was used to correct for multicollinarity. The residuals from a regression of FMI and FMI squared on FFMI were included in the models predicting the effect of FFMI on the levels of the markers, and vice versa for FMI. Thus, for each model we examined the effect of either FFMI or FMI, adjusted for the residuals of the other body composition index, as well as all mentioned potential confounders. Age, FEV1 \% pred, FFMI and FMI were all treated as continuous variables after testing the linearity assumption with the method of fractional polynomials [23].

Bioelectrical impedance was not measured in five patients: two patients had metal implants (pacemakers) and three patients refused measurement. The EIA analyses were performed before inclusion of seven patients, and blood sampling was unsuccessful in another six patients. For six patients we ran out of plasma before completing the analyses for either sTNF-R1 or OPG. Thus, the final multivariable models are based on 409 patients.

\section{RESULTS}

The baseline characteristics of the 409 COPD patients are given in table 1. Male patients were slightly older, had slightly lower FEV1 \% pred and had more comorbidity but were less likely to use inhaled steroids. Even though BMI was not significantly different between the sexes, FFMI was higher in males while FMI was higher in females. $31 \%$ of females had an FFMI 
$<14 \mathrm{~kg} \cdot \mathrm{m}^{-2}$ and $27.5 \%$ of males had an FFMI $<17 \mathrm{~kg} \cdot \mathrm{m}^{-2}$ (table 1). These FFMI cut-off values indicate the 5th percentile for FFMI in normal adult Caucasian male and female populations [24], and form a useful definition of cachexia [4].

When considered separately, a close relationship was found between FFMI and FMI in both males and females, as shown in figure 1. With increasing FFMI, there was an increase in FMI. However, the relationship differed somewhat between the sexes, where males had a narrower range of FMI for a wider range of FFMI (fig. 1).

\section{Association between markers of inflammation and body composition: bivariate analyses}

Mean plasma levels of CRP, sTNF-R1 and OPG by quartiles of FFMI, FMI and the FFMI/FMI ratio are shown in figures 2-4, respectively. CRP and sTNF-R1 increased significantly with increasing FFMI and also with increasing FMI (figs 2 and 3), whereas OPG decreased significantly with increasing FFMI,

\begin{tabular}{|c|c|c|c|}
\hline $\begin{array}{l}\text { Baseline characteri } \\
\text { obstructive pulmon }\end{array}$ & $\begin{array}{l}\text { ics of } 409 \\
y \text { disease }\end{array}$ & $\begin{array}{l}\text { chronic } \\
\text { (COPD) p }\end{array}$ & atients \\
\hline & Females & Males & p-value ${ }^{\#}$ \\
\hline Subjects $n$ & 160 & 249 & \\
\hline Age & $62.3 \pm 6.6$ & $64.2 \pm 7.0$ & $<0.01$ \\
\hline \multicolumn{4}{|l|}{ Body composition } \\
\hline $\mathrm{BMI} \mathrm{kg} \cdot \mathrm{m}^{-2}$ & $24.9 \pm 6.2$ & $25.7 \pm 4.9$ & 0.16 \\
\hline $\mathrm{FMI} \mathrm{kg} \cdot \mathrm{m}^{-2}$ & $10.1 \pm 4.3$ & $7.3 \pm 2.6$ & $<0.01$ \\
\hline $\mathrm{FFMI} \mathrm{kg} \cdot \mathrm{m}^{-2}$ & $14.8 \pm 2.4$ & $18.4 \pm 3.3$ & $<0.01$ \\
\hline Patients with pathologically low FFMI & 31.0 & 27.5 & 0.45 \\
\hline Smoking habits & & & 0.57 \\
\hline Ex-smoker & 55.0 & 57.8 & \\
\hline Current smoker & 45.0 & 42.2 & \\
\hline FEV $1 \%$ pred & $50.4 \pm 13.9$ & $47.8 \pm 14.4$ & 0.08 \\
\hline Hypoxaemia $\mathrm{Pa}, \mathrm{O}_{2}<8.0 \mathrm{kPa}^{+}$ & & & 0.31 \\
\hline No & 84.8 & 15.2 & \\
\hline Yes & 88.4 & 11.6 & \\
\hline Daily use of inhaled steroids & 75.0 & 64.7 & 0.03 \\
\hline Charlson Comorbidity Index & & & 0.01 \\
\hline 1 (COPD only) & 63.1 & 52.2 & \\
\hline 2 & 25.0 & 23.7 & \\
\hline 3 & 8.8 & 14.5 & \\
\hline $4-6$ & 3.1 & 9.6 & \\
\hline \multicolumn{4}{|l|}{ Inflammatory markers } \\
\hline $\mathrm{CRP} \mu \mathrm{g} \cdot \mathrm{mL}^{-1}$ & $8.9 \pm 12.5$ & $7.7 \pm 10.8$ & 0.78 \\
\hline $\mathrm{sTNF}-\mathrm{R} 1 \mathrm{pg} \cdot \mathrm{mL}^{-1}$ & $699 \pm 219$ & $758 \pm 250$ & 0.01 \\
\hline $\mathrm{OPG} n \mathrm{ng} \cdot \mathrm{mL}^{-1}$ & $6.0 \pm 2.1$ & $5.7 \pm 2.3$ & 0.16 \\
\hline
\end{tabular}

Data are presented as mean \pm SD or $\%$, unless otherwise stated. BMI: body mass index; FMI: fat mass index; FFMI: fat-free mass index; FEV1; forced expiratory volume in $1 \mathrm{~s}$; \% pred: \% predicted; $\mathrm{Pa}_{1} \mathrm{O}_{2}$ : arterial oxygen tension CRP: C-reactive protein; sTNF-R1; soluble tumour necrosis factor receptor 1 ; OPG: osteoprogerin. * : t-test for age, body composition, FEV1; Chi-squared for percentage of patients with low FFMI, smoking habits, use of inhaled steroids hypoxsemia and Charlson's comorbidity index; Wilcoxon Rank sum test for CRP, sTNF-R1 and OPG; ": $<14 \mathrm{~kg} \cdot \mathrm{m}^{-2}$ in females and $<17 \mathrm{~kg} \cdot \mathrm{m}^{-2}$ in males based on reference values from a normal population [24]; ${ }^{+}: 38$ subjects refused measurement or attempt was unsuccessful. but did not vary significantly with FMI (fig. 4). The analyses were also performed separately for females and males, but there was no significant sex difference in the relationship between the quartiles of FFMI and FMI and the three markers (data not shown).

To further attempt to disentangle the effects of FFMI and FMI, we looked at the association between FFMI and each of the three markers in males with an FMI $<10 \mathrm{~kg} \cdot \mathrm{m}^{-2}$ only. This was done in an attempt to separate the influences of FMI and FFMI on the biomarkers. The rationale was that, in this subgroup of subjects, there was a wide variation in FFMI, but only small variation in FMI (fig. 1). Thus, this group might provide the opportunity to better assess the effect of FFMI on the three markers. In this group, the correlation coefficient between FFMI and CRP was only $0.05(\mathrm{p}=0.5)$, that between FFMI and sTNF-R1 was only $0.02(\mathrm{p}=0.8)$, and that between FFMI and OPG was $-0.10(p=0.14)$. Thus, in this sub-sample there was no significant association between FFMI and the three markers. However, despite the small variation in FMI in this subsample, there was still a significant positive association between FMI and CRP (correlation coefficient 0.16; $p=0.02$ ) and between FMI and sTNF-R1 (correlation coefficient 0.22 $\mathrm{p}<0.01$ ), but no relationship between FMI and OPG (correlation coefficient $0.07 ; p=0.33$ )

\section{Association between markers of inflammation and body composition: multivariate analyses}

The effect (regression coefficients) of FFMI and FMI on the levels of the three markers CRP, sTNF-R1 and OPG in the COPD patients after adjustment for sex, age, smoking, FEV1, hypoxaemia, Charlson Comorbidity Index and use of inhaled steroids are shown in table 2.

In both models, adjustment was made for the other body composition index by way of residualisation, to reduce the effect of multicollinearity between FFMI and FMI.

For both CRP and sTNF-R1, the multivariate analyses confirmed the findings from the bivariate analyses. Higher FFMI and higher FMI were both associated with higher plasma levels of CRP and sTNF-R1 after adjustment for potential confounders. For OPG, the results were reversed compared with the bivariate analyses after adjustment for confounders. Higher FMI was significantly associated with lower levels of OPG. For FFMI, the association with OPG did not reach statistical significance.

\section{DISCUSSION}

In this cross-sectional study of $>400$ COPD patients, including $>100$ with cachexia, circulating plasma levels of the two inflammatory markers CRP and sTNF-R1 were not elevated in patients having lower FFMI, as noted both in the bivariate analyses, and in the multivariate analyses with adjustment for sex, age, smoking, FEV1, hypoxaemia, comorbidities, use of inhaled steroids and FMI. Rather, we found the exact opposite. Our analyses imply that fat mass in addition to fat-free mass was related to the plasma levels of CRP and sTNF-R1. In contrast to the pattern for CRP and sTNF-R1, low FMI was associated with high OPG levels, whereas no association was found between FFMI and OPG. 


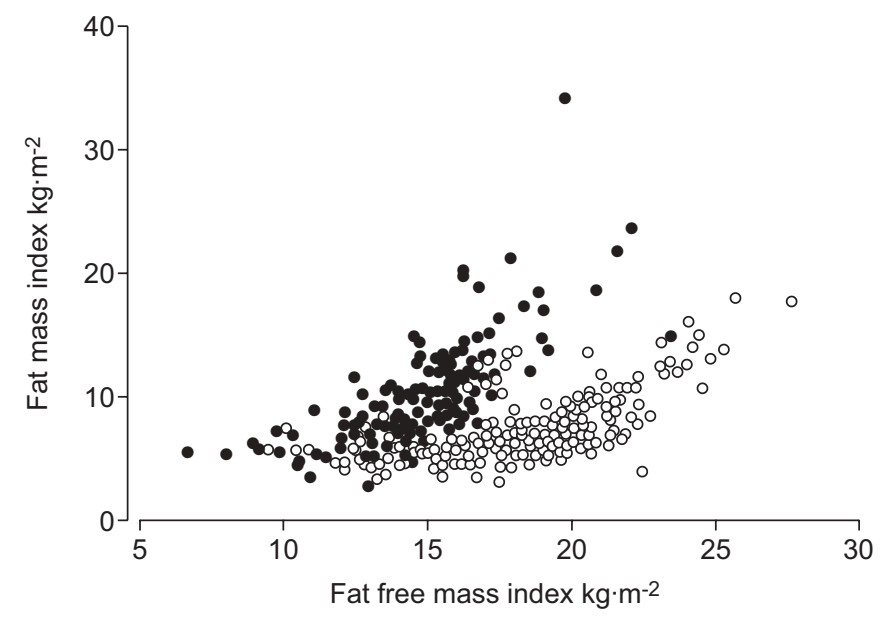

FIGURE 1. The distribution of fat mass index by fat free mass index in females (-) and males $(\bigcirc)$ in the baseline phase of the Bergen COPD Cohort Study.

COPD is a heterogeneous disease, with substantial individual variation in symptomatology, progression of lung function decline, exacerbation frequency and development of complications such as cachexia. Approximately 25\% of COPD patients develop cachexia [4], a serious complication associated with increased mortality within the COPD patient population. The reasons why some COPD patients develop cachexia and others do not remain elusive. One prominent theory suggested by several authors [25-27], is that pathological weight loss is at least partly explained by an increase in systemic inflammation reflected in enhanced plasma or serum levels of inflammatory markers.

Several cross-sectional studies have examined systemic levels of inflammatory markers in COPD patients with and without cachexia. Most of the studies have focused on TNF- $\alpha$ [7-10, 1214], and/or the TNF- $\alpha$ receptors [8, 9, 14, 17, 28-30], whereas two examined CRP $[15,31]$. The earliest published studies found elevated levels of TNF- $\alpha$ [7-10] in cahectic patients, whereas in later studies $[12,13]$, no such difference was found. In addition, over time the reported levels of TNF- $\alpha$ have decreased, from $6-70 \mathrm{pg} \cdot \mathrm{mL}^{-1}[7,8]$ in the earlier studies, to $0.5-1.2 \mathrm{pg} \cdot \mathrm{mL}^{-1}$ in the later studies $[12,13]$. This decrease probably reflects improvement in assay specificity over time, and suggests that group differences in the earliest studies should be confirmed by more specific assays.

Measurement of the two soluble TNF- $\alpha$ receptors, sTNF-R1 and STNF-R2, is easier than that for TNF- $\alpha$, in a large part because of much higher concentrations in serum and plasma. In addition, whereas TNF- $\alpha$ typically is pulse secreted, the receptors provide a more stable and reliable measurement of the activity of the TNF- $\alpha$ pathway [32]. In two previous studies that examined cachexia in COPD patients by systemic levels of the TNF- $\alpha$ receptors, one study found that the receptors were elevated in cachectic COPD patients compared with noncachectic COPD patients [9] and the other study did not [12]. However, the numbers of cachectic patients in these two studies were only 29 and 35, respectively.

Loss of muscle mass may not be the only factor when cachectic patients experience a loss of lean body mass, loss of bone mass may be important too. COPD patients with cachexia have an increased risk for developing osteoporosis [33]. OPG is a soluble member of the TNF receptor superfamily that inhibits osteoclastogenesis by binding the receptor activator of nuclear factor- $\kappa \mathrm{B}$ ligand (RANKL), acting as a decoy receptor to competitively inhibit RANKL interaction with its receptor RANK. However, while RANKL circulates in very low levels, OPG is a more stable overall measure of RANKL/RANK activity than RANKL, potentially reflecting inflammatory as well as bone and matrix remodelling activity induced by RANKL. We have previously shown that plasma levels of OPG were lower in COPD patients than subjects without COPD [16]. We are not aware of previous studies examining the relationship between systemic OPG and body composition. In the current study, high OPG was associated with low FMI, whereas no association was found between plasma levels of OPG and FFMI after adjustment for confounders. This finding could reflect a higher turnover of bone metabolism in COPD patients with low FMI, that OPG reflected inflammatory pathways not reflected by CRP or sTNF-R1, or a hitherto
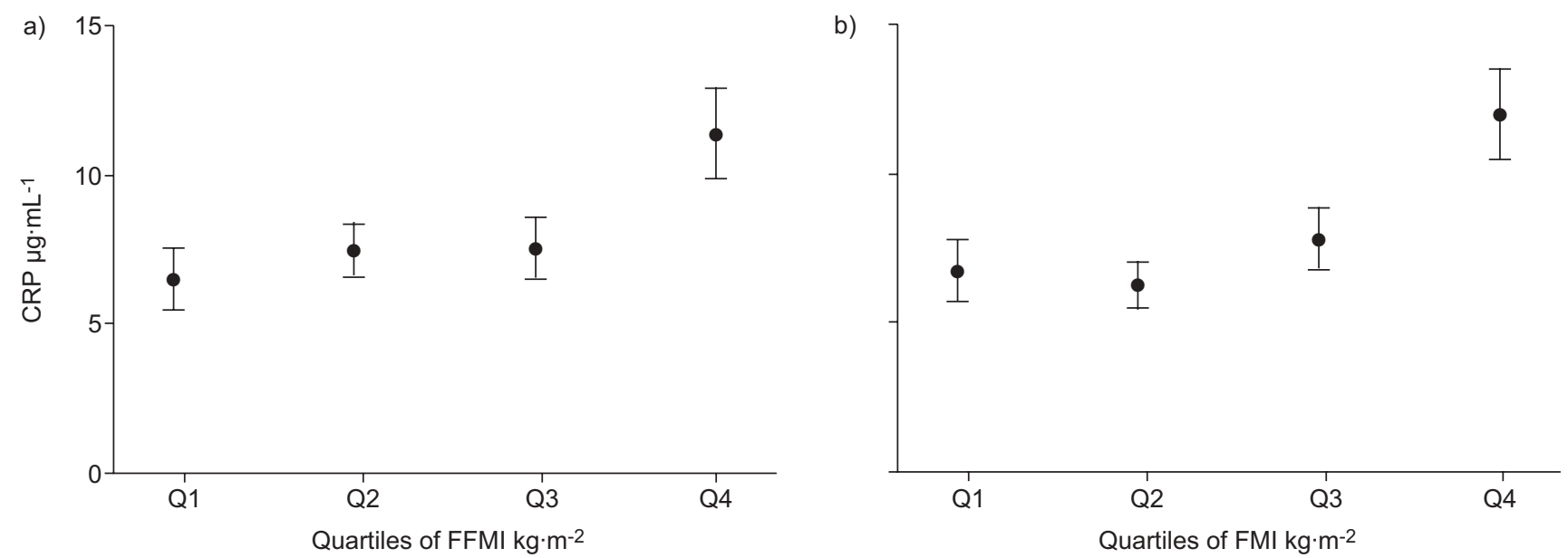

FIGURE 2. The mean \pm SE plasma level of C-reactive protein (CRP) by quartiles of a) fat-free mass index (FFMI) and b) fat mass index (FMI) among chronic obstructive pulmonary disease patients. $p<0.001$. 

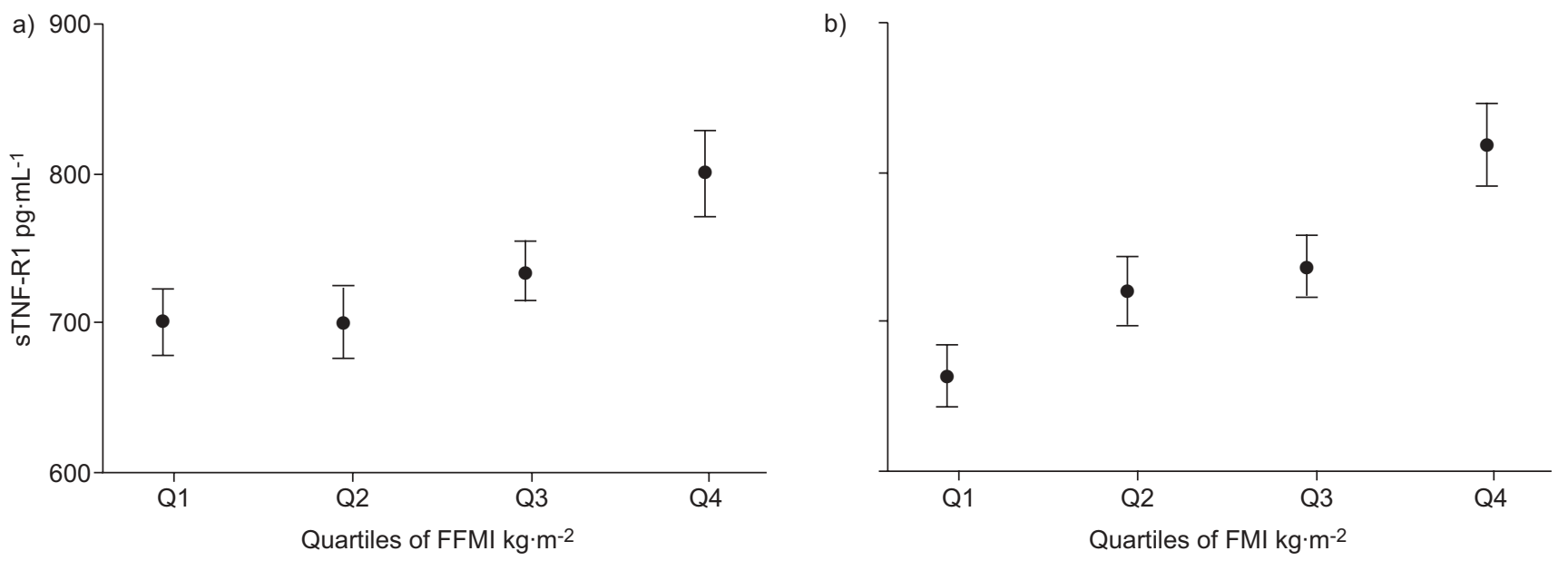

FIGURE 3. The mean \pm SE plasma level of soluble tumour necrosis factor receptor 1 (sTNF-R1) by quartiles of a) fat-free mass index (FFMI) and b) fat mass index (FMI) among chronic obstructive pulmonary disease patients. a) $p=0.008$, b) $p<0.001$.

unrecognised metabolic effect of the RANKL/OPG/RANK axis. Unfortunately, we did not have measurements of bone density in this study and, therefore, could not compare levels of OPG to degrees of measured osteoporosis. This could be an interesting topic for future studies.

The largest challenge in interpreting the results from the current study is the close relationship between FFMI and FMI. $\mathrm{BMI}$ is an imperfect tool in assessing body composition, as it does not separate lean body mass from fat mass and was, therefore, not used. Two subjects can vary widely in fat mass and still have the same BMI. To try to separate the effects of FFMI and FMI, we looked at the relationship between FFMI and the three markers in the subgroup of males with a FMI $<10 \mathrm{~kg} \cdot \mathrm{m}^{-2}$. In this group, where the variation of FFMI was great but the variation of FMI small, FFMI was not related to the levels of the three markers. In addition, the multivariable analyses indicated that both FFMI and FMI had independent effects on the levels of CRP and possibly sTNF-R1. Thus, these analyses support the conclusion that CRP and STNF-R1 is not elevated in cachectic COPD patients.

Our study highlights the probable contribution of metabolically active fat tissue in systemic inflammation in COPD. Adipose tissue may promote low-grade systemic inflammation and inflammation may promote dysfunction of adipocytes and adipose tissue. Previous studies have shown that overweight subjects have elevated CRP, both in healthy population studies [34] and in studies on other diseases, such as diabetes [35]. Furthermore, TNF- $\alpha$ has been shown to be produced by adipocytes, and its expression increases with adipocyte mass $[36,37]$. TNF- $\alpha$ increases insulin resistance and may be upregulated as a consequence of a high fat diet. Elevated levels of sTNF-R1 could be seen as a marker of this mechanism. There are not many studies looking specifically at obesity in COPD. However, in a small Canadian study on 28 COPD patients, overweight patients had higher systemic levels of CRP, TNF- $\alpha$ and IL-6 [38]. Thus, we suggest that inflammation
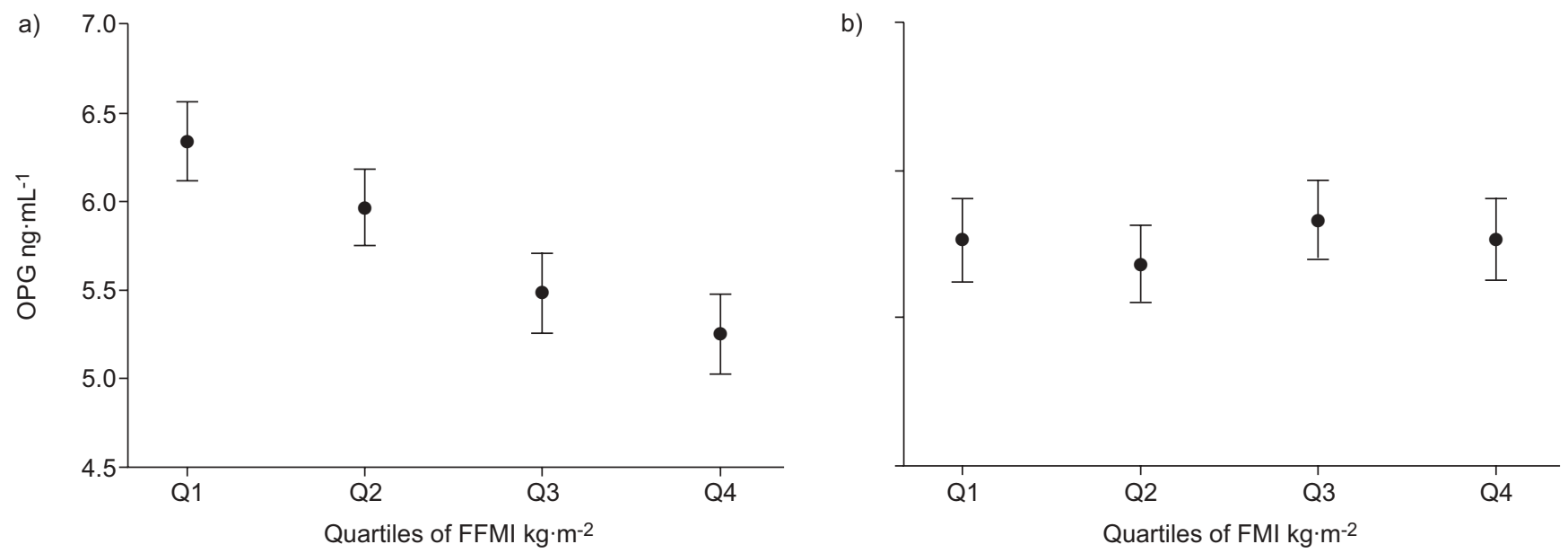

FIGURE 4. The mean \pm SE plasma level of osteoprotegerin (OPG) by quartiles of a) fat-free mass index (FFMI) and b) fat mass index (FMI) among chronic obstructive pulmonary disease patients. a) $p=0.008, b) p=0.9$. 


\begin{tabular}{|c|c|c|c|c|c|c|}
\hline \multirow[t]{2}{*}{ TABLE 2} & \multicolumn{6}{|c|}{$\begin{array}{l}\text { Regression coefficients from six multivariable } \\
\text { regression analyses assessing the association of } \\
\text { fat-free mass index (FFMI) and fat mass index } \\
\text { (FMI) to the level of three plasma markers }\end{array}$} \\
\hline & logCRP & $p$-value & sTNF-R1 & $p$-value & OPG & p-value \\
\hline FFMI $\mathrm{kg} \cdot \mathrm{m}^{-2}$ & $\begin{array}{c}1.23 \\
(1.14-1.33)\end{array}$ & $<0.01$ & $\begin{array}{c}24.9 \\
(11.8-38.1)\end{array}$ & $<0.01$ & $\begin{array}{c}-0.06 \\
(-0.19-0.06)\end{array}$ & 0.32 \\
\hline $\mathrm{FMI} \mathbf{k g} \cdot \mathrm{m}^{-2}$ & $\begin{array}{c}1.12 \\
(1.06-1.19)\end{array}$ & $<0.01$ & $\begin{array}{c}15.4 \\
(5.6-25.3)\end{array}$ & $<0.01$ & $\begin{array}{c}-0.14 \\
(-0.23--0.04)\end{array}$ & $<0.01$ \\
\hline \multicolumn{7}{|c|}{$\begin{array}{l}\text { Data are presented as regression coefficients ( } 95 \% \text { confidence interval), unless } \\
\text { otherwise stated. All models included sex, age, smoking, forced expiratory } \\
\text { volume in } 1 \mathrm{~s} \text {, Charlson Comorbidity Index, hypoxaemia, use of inhaled steroids } \\
\text { and the residuals from the other body composition index than the one in } \\
\text { question (see text for further details). CRP: C-reactive protein; STNF-R1: soluble } \\
\text { tumour necrosis factor receptor 1; OPG: osteoprotegerin. }\end{array}$} \\
\hline
\end{tabular}

in COPD may also be related to obesity within the COPD population, potentially contributing to the increased occurrence of obesity related comorbidities, such as coronary heart disease. This will need to be confirmed in longitudinal studies.

Some methodological shortcomings must be acknowledged. First, the study was cross-sectional. Therefore, there is the possibility that COPD patients prone to develop cachexia had higher systemic levels of inflammation prior to their loss of lean body mass, but as cachexia developed, levels of the markers fell. This can only be resolved in future longitudinal studies. Secondly, being an observational study we cannot say for certain whether the observed relationships are physiological or pathological. Thirdly, by design we only have COPD patients with global Initiative for Chronic Obstructive Lung Disease (GOLD) stage II or worse, and the results may not be applicable to patients with mild disease (GOLD stage I). Finally, we did not measure the levels of sTNF-R2 in the plasma of our patients. In previous studies on levels of TNF receptors in COPD [8, 9, 12, 14, 28-30], no real difference has been found between the two receptors, with regard to levels in COPD patients versus controls, and with regard to cachexia or no cachexia. However, whether that would also be true in the current cohort we cannot know. Ideally, future studies should include measurements of both TNF receptors and TNF- $\alpha$, as well as TNF- $\alpha$ bioactivity.

In conclusion, the current study does not support the theory that cachexia in COPD patients is mediated through an increase in TNF- $\alpha$ related systemic inflammation. Rather, the study results allow the hypothesis that high fat mass may also be a predictor of high plasma levels of CRP and sTNF-R1 in COPD patients. The relationship between FMI and OPG in COPD patients is novel, and could be an indication that fat metabolism is a factor in the development of osteoporosis in COPD patients. However, these findings need to be confirmed in longitudinal studies.

\section{SUPPORT STATEMENT}

The study was funded by grants from The Foundation for Respiratory Research (University of Bergen, Bergen, Norway) and from the Centre for Clinical Research, (Haukeland University Hospital, Bergen).

\section{STATEMENT OF INTEREST}

None declared.

\section{REFERENCES}

1 Halbert RJ, Natoli JL, Gano A, et al. Global burden of COPD: systematic review and meta-analysis. Eur Respir J 2006; 28: 523-532.

2 Calverley PM, Walker P. Chronic obstructive pulmonary disease. Lancet 2003; 362: 1053-1061.

3 Ferrer M, Alonso J, Morera J, et al. Chronic obstructive pulmonary disease stage and health-related quality of life. The Quality of Life of Chronic Obstructive Pulmonary Disease Study Group. Ann Intern Med 1997; 127: 1072-1079.

4 Wagner PD. Possible mechanisms underlying the development of cachexia in COPD. Eur Respir J 2008; 31: 492-501.

5 Schols AM, Broekhuizen R, Weling-Scheepers CA, et al. Body composition and mortality in chronic obstructive pulmonary disease. Am J Clin Nutr 2005; 82: 53-59.

6 Debigare $\mathrm{R}$, Cote $\mathrm{CH}$, Maltais F. Peripheral muscle wasting in chronic obstructive pulmonary disease. Clinical relevance and mechanisms. Am J Respir Crit Care Med 2001; 164: 1712-1717.

7 Di Francia M, Barbier D, Mege JL, et al. Tumor necrosis factoralpha levels and weight loss in chronic obstructive pulmonary disease. Am J Respir Crit Care Med 1994; 150: 1453-1455.

8 Takabatake N, Nakamura H, Abe S, et al. Circulating leptin in patients with chronic obstructive pulmonary disease. Am J Respir Crit Care Med 1999; 159: 1215-1219.

9 Eid AA, Ionescu AA, Nixon LS, et al. Inflammatory response and body composition in chronic obstructive pulmonary disease. Am J Respir Crit Care Med 2001; 164: 1414-1418.

10 Itoh T, Nagaya N, Yoshikawa M, et al. Elevated plasma ghrelin level in underweight patients with chronic obstructive pulmonary disease. Am J Respir Crit Care Med 2004; 170: 879-882.

11 Schols AM, Soeters PB, Dingemans AM, et al. Prevalence and characteristics of nutritional depletion in patients with stable COPD eligible for pulmonary rehabilitation. Am Rev Respir Dis 1993; 147: 1151-1156.

12 Broekhuizen R, Grimble RF, Howell WM, et al. Pulmonary cachexia, systemic inflammatory profile, and the interleukin 1beta -511 single nucleotide polymorphism. Am J Clin Nutr 2005; 82: 1059-1064.

13 Van Helvoort HA, Heijdra YF, Thijs HM, et al. Exercise-induced systemic effects in muscle-wasted patients with COPD. Med Sci Sports Exerc 2006; 38: 1543-1552.

14 Shin KC, Chung JH, Lee KH. Effects of TNF-alpha and leptin on weight loss in patients with stable chronic obstructive pulmonary disease. Korean J Intern Med 2007; 22: 249-255.

15 Kurosaki $\mathrm{H}$, Ishii $\mathrm{T}$, Motohashi $\mathrm{N}$, et al. Extent of emphysema on HRCT affects loss of fat-free mass and fat mass in COPD. Intern Med 2009; 48: 41-48.

16 Eagan TM, Ueland T, Wagner PD, et al. Systemic inflammatory markers in chronic obstructive pulmonary disease: results from the Bergen COPD Cohort Study. Eur Respir J 2010; 35: 540-548.

17 Broekhuizen R, Vernooy JH, Schols AM, et al. Leptin as local inflammatory marker in COPD. Respir Med 2005; 99: 70-74.

18 Horowitz MC, Xi Y, Wilson K, et al. Control of osteoclastogenesis and bone resorption by members of the TNF family of receptors and ligands. Cytokine Growth Factor Rev 2001; 12: 9-18.

19 Nabipour I, Kalantarhormozi M, Larijani B, et al. Osteoprotegerin in relation to type 2 diabetes mellitus and the metabolic syndrome in postmenopausal women. Metabolism 2010; 59: 742-747.

20 Johannessen A, Lehmann S, Omenaas ER, et al. Post-bronchodilator spirometry reference values in adults and implications for disease management. Am J Respir Crit Care Med 2006; 173: 1316-1325. 
21 Charlson ME, Pompei $\mathrm{P}$, Ales KL, et al. A new method of classifying prognostic comorbidity in longitudinal studies: development and validation. J Chronic Dis 1987; 40: 373-383.

22 Charlson M, Szatrowski TP, Peterson J, et al. Validation of a combined comorbidity index. J Clin Epidemiol 1994; 47: 1245-1251.

23 Royston P, Ambler G, Sauerbrei W. The use of fractional polynomials to model continuous risk variables in epidemiology. Int J Epidemiol 1999; 28: 964-974.

24 Schutz Y, Kyle UU, Pichard C. Fat-free mass index and fat mass index percentiles in Caucasians aged 18-98 y. Int J Obes Relat Metab Disord 2002; 26: 953-960.

25 Balasubramanian VP, Varkey B. Chronic obstructive pulmonary disease: effects beyond the lungs. Curr Opin Pulm Med 2006; 12: 106-112.

26 Barnes PJ, Celli BR. Systemic manifestations and comorbidities of COPD. Eur Respir J 2009; 33: 1165-1185.

27 Wouters EF, Creutzberg EC, Schols AM. Systemic effects in COPD. Chest 2002; 121: Suppl. 5, 127S-130S.

28 Bolton CE, Ionescu AA, Shiels KM, et al. Associated loss of fat-free mass and bone mineral density in chronic obstructive pulmonary disease. Am J Respir Crit Care Med 2004; 170: 1286-1293.

29 Cohen RI, Marzouk K, Berkoski P, et al. Body composition and resting energy expenditure in clinically stable, non-weight-losing patients with severe emphysema. Chest 2003; 124: 1365-1372.

30 Schols AM, Buurman WA, Staal van den Brekel AJ, et al. Evidence for a relation between metabolic derangements and increased levels of inflammatory mediators in a subgroup of patients with chronic obstructive pulmonary disease. Thorax 1996; 51: 819-824.
31 Pinto-Plata VM, Mullerova H, Toso JF, et al. C-reactive protein in patients with COPD, control smokers and non-smokers. Thorax 2006; 61: 23-28.

32 Dempsey PW, Doyle SE, He JQ, et al. The signaling adaptors and pathways activated by TNF superfamily. Cytokine Growth Factor Rev 2003; 14: 193-209.

33 Graat-Verboom L, Spruit MA, van den Borne BE, et al. Correlates of osteoporosis in chronic obstructive pulmonary disease: An underestimated systemic component. Respir Med 2009; 103: 1143-1151.

34 Nguyen XM, Lane J, Smith BR, et al. Changes in inflammatory biomarkers across weight classes in a representative US population: a link between obesity and inflammation. J Gastrointest Surg 2009; 13: 1205-1212.

35 Hofso D, Ueland $\mathrm{T}$, Hager $\mathrm{H}$, et al. Inflammatory mediators in morbidly obese subjects: associations with glucose abnormalities and changes after oral glucose. Eur J Endocrinol 2009; 161: 451-458.

36 Kern PA, Saghizadeh M, Ong JM, et al. The expression of tumor necrosis factor in human adipose tissue. Regulation by obesity, weight loss, and relationship to lipoprotein lipase. J Clin Invest 1995; 95: 2111-2119.

37 Skolnik EY, Marcusohn J. Inhibition of insulin receptor signaling by TNF: potential role in obesity and non-insulin-dependent diabetes mellitus. Cytokine Growth Factor Rev 1996; 7: 161-173.

38 Poulain M, Doucet M, Drapeau V, et al. Metabolic and inflammatory profile in obese patients with chronic obstructive pulmonary disease. Chron Respir Dis 2008; 5: 35-41. 